\title{
Establishing an Operational Definition of Sarcopenia in Australia and New Zealand: Delphi Method Based Consensus Statement
}

Zanker, J.; Scott, D.; Reijnierse, E. M.; Brennan-Olsen, S. L.; Daly, R. M.; Girgis, C. M.;

Grossmann, M.; Hayes, A.; Henwood, T.; Hirani, V.; Inderjeeth, C. A.; Iuliano, S.; Keogh, J. W.L.; Lewis, J. R.; Maier, A. B.; Pasco, J. A.; Phu, S.; Sanders, K. M.; Sim, M.; Visvanathan, R.; Waters, D. L.; Yu, S. C.Y.; Duque, Gustavo

Published in:

Journal of Nutrition, Health and Aging

DOI:

$10.1007 / \mathrm{s} 12603-018-1113-6$

Licence:

Unspecified

Link to output in Bond University research repository.

Recommended citation(APA):

Zanker, J., Scott, D., Reijnierse, E. M., Brennan-Olsen, S. L., Daly, R. M., Girgis, C. M., Grossmann, M., Hayes, A., Henwood, T., Hirani, V., Inderjeeth, C. A., luliano, S., Keogh, J. W. L., Lewis, J. R., Maier, A. B., Pasco, J. A., Phu, S., Sanders, K. M., Sim, M., ... Duque, G. (2019). Establishing an Operational Definition of Sarcopenia in Australia and New Zealand: Delphi Method Based Consensus Statement. Journal of Nutrition, Health and Aging, 23(1), 105-110. https://doi.org/10.1007/s12603-018-1113-6

\section{General rights}

Copyright and moral rights for the publications made accessible in the public portal are retained by the authors and/or other copyright owners and it is a condition of accessing publications that users recognise and abide by the legal requirements associated with these rights.

For more information, or if you believe that this document breaches copyright, please contact the Bond University research repository coordinator. 


\section{Establishing an Operational Definition of Sarcopenia in Australia and New Zealand:}

\section{Delphi Method Based Consensus Statement}

Jesse Zanker ${ }^{1,2}$, David Scott ${ }^{1,2,3}$, Esmee M. Reijnierse ${ }^{4}$, Sharon L. Brennan-Olsen ${ }^{1,2}$,

Robin M. Daly ${ }^{5}$, Christian M. Girgis ${ }^{6}$, Mathis Grossmann ${ }^{7}$, Alan Hayes ${ }^{1,2,8}$, Tim

Henwood ${ }^{9,10}$, Vasant Hirani ${ }^{11-13}$, Charles A. Inderjeeth ${ }^{14}$, Sandra Iuliano ${ }^{7}$, Justin W.L.

Keogh ${ }^{15-17}$, Joshua Lewis ${ }^{18,19}$, Andrea B. Maier ${ }^{4,20}$, Julie A. Pasco ${ }^{2,21,22}$, Steven Phu ${ }^{1,2}$,

Kerrie M. Sanders ${ }^{2}$, Marc Sim ${ }^{18}$, Renuka Visvanathan ${ }^{23,24}$, Debra L. Waters ${ }^{25}$, Solomon

C.Y. Yu ${ }^{23,24}$, Gustavo Duque ${ }^{1,2}$

On behalf of the Australian and New Zealand Society for Sarcopenia and Frailty

Research Task Force on Diagnostic Criteria for Sarcopenia

${ }^{1}$ Australian Institute for Musculoskeletal Science (AIMSS), The University of

Melbourne and Western Health, St. Albans, Victoria, Australia

${ }^{2}$ Department of Medicine - Western Health, The University of Melbourne, St. Albans,

Victoria, Australia

${ }^{3}$ Department of Medicine, School of Clinical Sciences at Monash Health, Monash

University, Clayton, Victoria, Australia

${ }^{4}$ Department of Medicine and Aged Care, @AgeMelbourne, The Royal Melbourne

Hospital, The University of Melbourne, Parkville, Victoria, Australia

${ }^{5}$ Institute for Physical Activity and Nutrition, Deakin University, Geelong, Melbourne, Victoria, Australia

${ }^{6}$ Departments of Endocrinology, Royal North Shore and Westmead Hospitals, University of Sydney, New South Wales, Australia 
${ }^{7}$ Department of Medicine, The University of Melbourne, Department of

Endocrinology, Austin Health, Victoria, Australia

${ }^{8}$ Institute for Health and Sport, Victoria University, Footscray, Victoria, Australia

${ }^{9}$ Southern Cross Care South Australia and Northern Territory, Australia

${ }^{10}$ Human Movement and Nutritional Science, University of Queensland, Brisbane,

Queensland, Australia

${ }^{11}$ Nutrition and Dietetics Group, School of Life and Environmental Sciences Charles

Perkins Centre, University of Sydney, New South Wales, Sydney, Australia

${ }^{12}$ Centre for Education and Research on Ageing, University of Sydney and Ageing,

Ageing and Alzheimer's Institute, Ageing and Alzheimer's Institute, Concord Hospital,

New South Wales, Sydney, Australia

${ }^{13}$ The ARC Centre of Excellence in Population Ageing Research (CEPAR), University of

New South Wales, New South Wales, Sydney, Australia

${ }^{14}$ North Metropolitan Health Service \& University of Western Australia, Perth,

Western Australia, Australia

${ }^{15}$ Faculty of Health Sciences and Medicine, Bond University, Queensland, Australia

${ }^{16}$ Human Potential Centre, AUT University, Auckland, New Zealand

${ }^{17}$ Cluster for Health Improvement, Faculty of Science, Health, Education and

Engineering, University of the Sunshine Coast, Queensland, Australia

${ }^{18}$ School of Medical and Health Sciences, Edith Cowan University, Joondalup, Perth, Western Australia, Australia

${ }^{19}$ School of Public Health, University of Sydney, Sydney, New South Wales, Australia

${ }^{20}$ Department of Human Movement Sciences, @AgeAmsterdam, Amsterdam

Movement Sciences, Vrije Universiteit, Amsterdam, The Netherlands 
${ }^{21}$ Epi-Centre for Healthy Ageing, Deakin University, Geelong, Victoria, Australia

22 Department of Epidemiology and Preventive Medicine, Monash University, Melbourne, Victoria, Australia

${ }^{23}$ Aged \& Extended Care Services, Medical Directorate, The Queen Elizabeth Hospital, Central Adelaide Local Health Network, South Australia, Australia

${ }^{24}$ National Health and Medical Council Centre of Research Excellence Trans-

Disciplinary Frailty Research To Achieve Health Aging and the Adelaide Geriatrics Training and Research with Aged Care (GTRAC) Centre, School of Medicine, Faculty of Health Sciences, University of Adelaide, South Australia, Australia

${ }^{25}$ University of Otago, Department of Medicine and School of Physiotherapy, Dunedin, New Zealand

\section{Corresponding author}

Prof. Gustavo Duque, MD, PhD, FRACP, FGSA

Australian Institute for Musculoskeletal Science (AIMSS)

176 Furlong Road, St. Albans, VIC, Australia 3021

e-mail: gustavo.duque@unimelb.edu.au

phone: +61 83958121

\section{Key Words}

Sarcopenia, Definition, Consensus, Delphi

\section{Funding}


This research did not receive any funding from agencies in the public, commercial, or not-for-profit sectors.

\section{Declarations}

Within the last five years, RV has attended advisory or consensus meetings, received grants and honorarium as well as presented for Nestle, Nutricia and Abbott. 


\section{Abstract}

\section{Background}

Globally there are several operational definitions for sarcopenia, complicating clinical and research applications.

\section{Aim}

The aim of the Australian and New Zealand Society for Sarcopenia and Frailty Research (ANZSSFR) Task Force on Diagnostic Criteria for Sarcopenia was to reach consensus on the operational definition of sarcopenia for regional use by clinicians and researchers.

\section{Method}

A four-Phase modified Delphi process was undertaken in which 24 individuals with expertise or a recognised interest in sarcopenia from different fields across Australia and New Zealand were invited to be Task Force members. An initial face-to-face meeting was held in Adelaide, South Australia, in November 2017, followed by two subsequent online Phases conducted by electronic surveys. A final Phase was used to approve the final statements. Responses were analysed using a pre-specified strategy. The level of agreement required for consensus was $80 \%$.

\section{Results}

In Phase 2, 94.1\% of Task Force respondents voted in favour of adopting an existing operational definition of sarcopenia. In Phase 3, $94.4 \%$ of respondents voted in 
favour of adopting the European Working Group on Sarcopenia in Older People

(EWGSOP) definition as the operational definition for sarcopenia in Australia and

New Zealand.

\section{Conclusion}

With consensus achieved, the ANZSSFR will adopt, promote and validate the

EWGSOP operational definition of sarcopenia for use by clinicians and researchers in Australia and New Zealand. 


\section{Introduction}

Sarcopenia is defined as an age-related disease of low muscle mass and low muscle strength or function [1]. Sarcopenia is of increasing clinical importance due to growing evidence of its health implications and the increasing proportion of older people in the population. Sarcopenia has been associated with an increased risk and rate of falls [2], fractures [3], functional impairment [2], metabolic syndrome [4], hospital admission and readmission [2], [5] poorer prognosis in cancer [6] and liver cirrhosis [7], surgical morbidity [8] and all-cause mortality [2], [6]. The clinical significance of sarcopenia as a distinct disease entity was established with the assignment of an International Classification of Diseases, Tenth Revision, Clinical Modification (ICD-10-CM) code (M62.84) in September 2016 [9].

A 2017 systematic review and meta-analysis including 35 studies with 58,404 individuals estimated that the prevalence of sarcopenia globally was $10 \%$ in both men and women, and higher among non-Asian than Asian individuals [10]. Others have reported the prevalence of sarcopenia to range from 1-29\% in communitydwelling populations, $14-33 \%$ in long-term care populations and $10 \%$ in acute hospital settings [11]. This marked heterogeneity is largely due to the different definitions applied to diagnose sarcopenia [12]. Nevertheless, as the global population ages, the number of sarcopenic older adults is projected to increase substantially over the coming decades. As such, research is needed to help alleviate the burden of sarcopenia. For this to be effective, a clear consensus of what defines sarcopenia needs to be established. 
Several operational definitions of sarcopenia have been developed [1], [13] - [17]

since the term was first coined almost 30 years ago [18]. The most widely used operational definitions, combining both low muscle mass and function, are described in Appendix 1. While there is widespread agreement on sarcopenia as a disease entity, there is no uniform consensus regarding the operational definition of sarcopenia. This leads to challenges in comparing results from research studies utilising different definitions of sarcopenia, and undoubtedly is a source of confusion for both researchers and clinicians. Indeed, only one in five health care professionals know how to diagnose sarcopenia using the operational definitions available [19].

The most widely utilised consensus definition of sarcopenia in the research literature was developed by the European Working Group on Sarcopenia in Older People (EWGSOP) in 2010 [1]. The EWGSOP definition includes an algorithm comprising gait speed, handgrip strength and muscle mass with cut-points dependent upon the individual's demographics (sex and height) [1]. In 2014, this definition was modified and validated by the Asian Working Group on Sarcopenia in recognition of the impact ethnic differences have on cut-points for muscle mass, strength and performance [18].

The establishment of a demographically-appropriate operational definition of sarcopenia is required to unify researchers and provide clear guidance to clinicians for the diagnosis and treatment of sarcopenia [20]. A group of individuals with an interest or expertise in sarcopenia were invited to join a Task Force and participate in a consensus-building collaboration with the aim of establishing an operational 
definition of sarcopenia for use by researchers and clinicians in Australia and New Zealand.

\section{Methods}

A four-Phase modified Delphi method was employed to achieve consensus amongst Task Force members on the preferred operational definition of sarcopenia for use in Australia and New Zealand (Figure 1). The Delphi method supports the structuring of group communication to allow a group of individuals to, as a collective, deal with complex problems and reach consensus [21]. The modified Delphi method [22] employed by the Task Force allowed for face-to-face communication in Phase 1 and approval of final statements in Phase 4. The Task Force leaders (GD, DS, JZ) designed and modified the questionnaires in Phases 2 and 3. The modified Delphi method used by the Australian and New Zealand Society for Sarcopenia and Frailty Research (ANZSSFR) Task Force adhered to preferred reporting methods and procedures [23].

\section{Selection of Task Force members}

A group of individuals with an interest or expertise in sarcopenia (physicians, geriatricians, primary and post-doctoral researchers, allied health professionals, health service managers) were invited to participate in a focus group discussion and form a Task Force to achieve consensus on the operational definition of sarcopenia in Australia and New Zealand. Prior to the first meeting, participants were provided with an overview, background, aims, strategies and 17 key references related to the different operational definitions of sarcopenia (Appendix 2). 
Phase 1 - Face-to-face meeting

On November $26^{\text {th }}, 2017$ following the ANZSSFR Scientific Meeting in Adelaide, Australia, 19 of the 24 invited Task Force members met for a four-hour meeting. Two presentations were delivered by researchers to the Task Force (DS and EMR) who summarised the key issues and outlined the agenda. Two focus groups were formed with key discussion points including whether the Task Force should adopt an existing definition or establish a new definition of sarcopenia, and to identify knowledge gaps in sarcopenia research. The de-identified minutes of the meeting are attached as Appendix 3 and informed the Second Round Questionnaire (2RQ).

\section{Phase 2 - Online Questionnaire}

On December 20 th 2017 an online, anonymous questionnaire (2RQ) developed by GD, DS and JZ was circulated to 23 Task Force members. The minutes of Phase 1 were provided to Task Force members. The pre-specified level of agreement (80\%) required for consensus was selected by the Task Force Leaders based on recommended practice [24], [25] and comparable modified Delphi studies [26]. The methodology was outlined to Task Force members who were given three weeks to complete the $2 \mathrm{RQ}$. A total of 12 questions and statements were contained in the $2 R Q$, which included four statements or key questions, four demographic questions and four free-text questions, attached as Appendix 4. The findings of the $2 R Q$ informed the development of the Third Round Questionnaire (3RQ).

\section{Phase 3-Online Questionnaire}


On February $26^{\text {th }}, 2018$ an online, anonymous questionnaire (3RQ) informed by the $2 \mathrm{RQ}$ and developed by GD, DS and JZ was circulated to 24 Task Force members who were given three weeks to complete the $3 R Q$. The results of the $2 R Q$ were provided verbatim to Task Force members. A total of three statements and questions and four demographic questions were included in the $3 R Q$ (Appendix 5).

\section{Phase 4-Circulation of position paper}

The results of the $3 R Q$ were provided verbatim to Task Force members. The statements and questions accepted to the pre-specified level of agreement in Phases 2 and 3 were circulated amongst Task Force members. Task Force members were requested to vote on a single statement contained within the position paper.

\section{Statistical Analysis}

Statistical analysis was undertaken using the pre-specified level of agreement (>80\%) required to accept a statement. Statements or questions that did not reach a level of agreement greater than $80 \%$ but achieved moderate agreement $(70-80 \%)$ in $2 R Q$ were re-examined in $3 R Q$, consistent with comparable modified Delphi methodologies [26]. Due to the size of the Task Force in addition to the small number of questions and statements being examined, investigation of dispersion or heterogeneity was not undertaken as is often performed in larger modified Delphi method studies [26]. 


\section{Results}

A total of 19/24 Task Force members participated in Phase 1. Due to one drop-out from Phase 1, 23 Task Force members were surveyed in Phase 2. In Phase 3, 24 individuals were surveyed following the re-joining of a Task Force member. Response rates in Phases $2(n=17,73.9 \%)$ and $3(n=18,75.0 \%)$ were similar. Due to de-identification, it is unknown whether there was consistency in responding Task Force members in Phases 2 and 3. The demographic details of participating Task Force members in Phases 2 and 3 are illustrated in Table 1.

In total, two statements regarding the operational definition of sarcopenia were accepted to a level greater than $80 \%$ and five were rejected (28.6\% acceptance rate). Questions, statements and respective levels of agreement in each Phase are listed in Table 2. In Phase 2, 94.1\% of respondents agreed that "an existing operational definition for sarcopenia should be adopted and validated using existing Australian and New Zealand data sets." Agreement on a preferred definition of sarcopenia was moderate in Phase 2 , with $70.6 \%$ of respondents supporting the EWGSOP definition [1], 17.6\% supporting the Foundation for the National Institute of Health Biomarkers Consortium Sarcopenia Project (FNIH) definition [16], and $11.8 \%$ supporting other [13] or new definitions. Consensus was not achieved (76.5\%) on the adoption and promotion of the sarcopenia screening tool, SARC-F [27], for use by primary care clinicians.

In Phase 3, 94.4\% of respondents agreed to adopt the EWGSOP operational definition of sarcopenia. Consensus was not achieved on whether the ANZSSFR 
should adopt and promote the accepted operational definition in its current form, including established cut-points, or await validation studies among Australian and New Zealand populations. There was moderate agreement (72.2\%) amongst respondents that an established definition should be adopted and promoted immediately, with cut-points for sarcopenia components (e.g. muscle mass, muscle strength and gait speed) modified should future validation studies suggest this is necessary for Australian and New Zealand populations. Conversely, 22.2\% of respondents agreed that the promotion of an established definition should await validation studies, while $5.6 \%$ of respondents agreed that an existing definition including cut-points should be adopted and promoted in its entirety without need for further validation among Australian and New Zealand populations.

In Phase 4, Task Force members were presented with a position paper and surveyed on their agreement with the statement posed in 3RQ, that the ANZSSFR should adopt and promote the EWGSOP and its established cut-points and modify these cut-points if validation studies suggest this as necessary. There was $100 \%$ agreement from 23 respondents to this statement.

\section{Discussion}

To date, a definition of sarcopenia has not been specifically developed for, or validated in, Australian and New Zealand populations. Across the globe, demographic differences have resulted in various measurement cut-points [17], therefore the ANZSSFR Task Force on Sarcopenia was formed to achieve consensus on an operational definition of sarcopenia for use by researchers and clinicians in 
Australia and New Zealand. Leading researchers in sarcopenia have called for a consensus definition [20], a sentiment echoed in Phase 1 of the Delphi method undertaken by the Task Force. Task Force members achieved consensus in Phase 2 of the Delphi method to adopt and validate an existing definition of sarcopenia, rather than develop a new definition in Australia and New Zealand. The consensus to adopt the EWGSOP operational definition of sarcopenia reflects the fact that this is currently the most cited definition of sarcopenia worldwide [1].

While consensus was achieved on the adoption of the EWGSOP operational definition of sarcopenia, the timing of when to promote the definition fell short of the pre-specified level of agreement in Phase 3. A majority of respondents $(72.2 \%)$ in Phase 3 agreed that the EWGSOP definition should be promoted immediately and subsequent validation studies should determine whether diagnostic cut-points need to be modified. Consensus on this statement was achieved in Phase 4 with agreement of $100 \%$. Therefore the ANZSSFR Task Force have achieved consensus for the immediate promotion of the EWGSOP definition in its activities directed at researchers and clinicians. A key objective for the Task Force will be to determine whether appropriate cut-points for muscle mass, handgrip strength and gait speed can be established using data from local cohort studies if the EWGSOP cut-points are found not to be predictive of sarcopenia-related outcomes in Australian and New Zealand older adult populations [1]. Australia and New Zealand are comprised of ethnically diverse, indigenous and immigrant populations to whom existing sarcopenia definitions and cut-points may not apply. The Asian Working Group on Sarcopenia previously adopted modified cut-points for the EWGSOP definition 
reflective of differences in muscle mass and strength observed between Asian and European older adults [18]. Our Task Force will also promote sarcopenia research in Australia and New Zealand's diverse populations to determine whether different cutpoints are required in different ethnic groups within our countries. Further, if these validation studies or further research in sarcopenia suggest other measures of muscle mass, muscle strength and physical performance are superior to those adopted, additional examination of the definition by the Task Force may be required.

In clinical practice, diagnostic tools such as dual energy X-ray absorptiometry (DXA), bioimpedance analysis (BIA) and handgrip strength dynamometers may not be immediately available to the clinician. A screening tool for sarcopenia, SARC-F, was developed for use by clinicians and was found to be valid for predicting adverse outcomes in sarcopenia [27]. A recent meta-analysis demonstrated that the SARC-F had poor sensitivity but high specificity for predicting those that should undergo further diagnostic testing for sarcopenia [28]. While the majority (61.1\%) of respondents reported a decision should be made on the use of SARC-F following further research, only $33.3 \%$ supported the immediate adoption and promotion of this tool for use as a screening device in the Australia and New Zealand. Therefore, the Task Force will advocate for further research on SARC-F in Australia and New Zealand and re-evaluate support for the SARC-F as a screening tool in the future.

A Delphi method may be limited by the breadth and diversity of its participants and rate of responses. In this modified Delphi method, a broad range of Task Force members across Australia and New Zealand were involved, however this process was 
limited by low or no representation from some Australian states. This Delphi method was strengthened by response rates in Phases 2, 3 and 4 exceeding $70 \%$ and the release of results verbatim to Task Force members. While preference exists for the involvement of patients or consumers in a Delphi method [23], the complexity and depth of knowledge required to judiciously answer questions and statements required experts in the field of sarcopenia, particularly given the low levels of public awareness of sarcopenia. Nevertheless, future activities for this Task Force will seek to engage clinicians and consumers.

The development of this consensus definition has the potential to unify researchers across Australia and New Zealand and assist with further international collaborations. In addition, the provision of a consistent definition and message to primary care clinicians and the aged care and long-term care sectors, where prevalence is at its highest, may increase diagnosis and treatment of sarcopenia.

\section{Conclusion}

The ANZSSFR will adopt and promote the EWGSOP operational definition of sarcopenia in Australia and New Zealand however the ANZSSFR acknowledges further research is required to validate the definition in this setting. This is a significant step towards regional and international consensus on sarcopenia in the understanding of this disease. Validation studies will be undertaken using this definition among specific regional and ethnic populations, and may result in future recommendations which provide adapted cut-points for individual components of sarcopenia. Any future variations to the current EWGSOP definition will be 
considered and evaluated by the ANZSSFR Task Force prior to adoption and validation.

\section{References}

1. Cruz-Jentoft AJ, Baeyens JP, Bauer JP, et al. Sarcopenia: European consensus on definition and diagnosis: Report of the European working group on sarcopenia in older people. Age Ageing. 2010;39(4)412-23. DOI 10.1093/ageing/afq034

2. Beaudar C, Zaaria M, Pasleau F, et al. Health outcomes of sarcopenia: A systematic review and meta-analysis. PloS One. 2017;12(1)e0169548. 10.1371/journal.pone.0169548

3. Zhang $\mathrm{Y}, \mathrm{Hao} \mathrm{Q}, \mathrm{Ge} \mathrm{M}$, Dong B. Association of sarcopenia and fractures in community-dwelling older adults: A systematic review and meta-analysis of cohort studies. Osteoporos Int. 2018 DOI 10.1007/s00198-018-4429-5 [ePub ahead of print].

4. Zhang $\mathrm{H}$, Lin $\mathrm{S}$, Gao T, et al. Association between sarcopenia and metabolic syndrome in middle-aged and older non-obese adults: A systematic review and meta-analysis. Nutrients. 2018;10:3. DOI 10.3390/nu10030364

5. Zhao Y, Zhang Y, Hao Q, et al. Sarcopenia and hospital-related outcomes in the old people: A systematic review and meta-analysis. Aging Clin Exp Res. 2018. DOI 10.1007/s40520-018-0931-z [ePub ahead of print].

6. Shachar SS, Williams GR, Muss HB, Nishijima TF. Prognostic value of sarcopenia in adults with solid tumours: A meta-analysis and systematic review. Eur J Cancer. 2016;57:58-67. DOI 10.1016/j.ejca.2015.12.030. 
7. Kim G, Kang SH, Kim MY, Baik SK. Prognostic value of sarcopenia in patients with liver cirrhosis: A systematic review and meta-analysis. PloS One. 2017;12:10(e0186990). DOI 10.1371/journal.pone.0186990

8. Jones K, Gordon-Weeks A, Coleman C, Silva M. Radiologically determined sarcopenia predicts morbidity and mortality following abdominal surgery: A systematic review and meta-analysis. World J Surg. 2017;41(9):2266-2279. DOI 10.1007/s00268-017-3999-2.

9. Anker SD, Morley JE, von Haehling S. Welcome to the ICD-10 code for sarcopenia. J Cachexia Sarcopenia Muscle. 2016;7(5):512-514. DOI $10.1002 / j c s m .12147$

10. Shafiee G, Keshtkar A, Soltani A, et al. Prevalence of sarcopenia in the world: a systematic review and meta- analysis of general population studies. J Diabetes Metab Disord. 2017; 16:21.

11. Cruz-Jentoft AJ, Landi F, Schneider SM, et al. Prevalence of and interventions for sarcopenia in ageing adults: A systematic review. Report of the international sarcopenia initiative (EWGSOP and IWGS). Age Ageing. 2014;43(6):748-59. DOI 10.1093/ageing/afu115

12. Reijnierse EM, Trappenburg MC, Leter MJ, et al. The impact of different diagnostic criteria on the prevalence of sarcopenia in healthy elderly participants and geriatric outpatients. Gerontol. 2015;61(6):491-6. DOI

\section{$10.1159 / 000377699$}

13. Baumgartner RN, Koehler KM, Gallagher D, et al. Epidemiology of sarcopenia among the elderly in New Mexico. Am J Epidemiol 1998; 147(8):755-63. 
14. Fielding RA, Vellas B, Evans WJ, et al. Sarcopenia: An undiagnosed condition in older adults. Current consensus definition: Prevalence, etiology, and consequences. International working group on sarcopenia. J Am Med Dir Assoc. 2011;12(4):249-56. DOI 10.1016/j.jamda.2011.01.003

15. Morley JE, Abbatecola AM, Argiles JM, et al. Sarcopenia with limited mobility: An international consensus. J Am Med Dir Assoc. 2011;12(6):403-9. DOI 10.1016/j.jamda.2011.04.014

16. Studenski SA, Peters KW, Alley DE, et al. The FNIH sarcopenia project: rationale, study description, conference recommendations, and final estimates. J Gerontol A Biol Sci Med Sci. 2014;69(5):547-58. DOI 10.1093/gerona/glu010

17. Chen LK, Liu LK, Woo J, et al. Sarcopenia in Asia: consensus report of the Asian Working Group for Sarcopenia. J Am Med Dir Assoc. 2014;15(2):95-101.

18. Rosenberg IH, Summary comments. Am J Clin Nutr. 1989;50(5):1231-1233.

19. Reijnierse EM,de van der Schueren MAE, Trappenburg MC, et al. Lack of knowledge and availability of diagnostic equipment could hinder the diagnosis of sarcopenia and its management. PloS One. 2017:12(10) e0185837. DOI 10.1371/journal.pone.0185837

20. Bijlsma AY, Meskers CG, Westendorp RG, Maier AB. Chronology of agerelated disease definitions: Osteoporosis and sarcopenia. Ageing Res Rev. 2012;11(2):320-4. DOI 10.1016/j.arr.2012.01.001

21. Linstone HA, Turoff M. The delphi method: Techniques and applications. Wesley Publishing Company, 2002. 
22. Temkin-Greener H, Ladwig S, Caprio T, et al. Developing palliative care practice guidelines and standards for nursing home-based palliative care teams: a Delphi study. J Am Med Dir Assoc. 2015;16(1):86.e1-7.

23. Boulkedid R, Abdoul H, Loustau M, et al. Using and reporting the delphi method for selecting healthcare quality indicators: A systematic review. PloS One. 2011;6(6)e20476. DOI 10.1371/journal.pone.0020476

24. Ulschak F. Human resource development: The theory and practice of need assessment. Reston, VA: Reston Publishing Company, Inc. 1983

25. Hsu C, Sandford B. The delphi technique: Making sense of consensus. Prac Assess Res Eval. 2007;12:1-8.

26. Rodriguez-Manas L, Feart C, Mann G, et al. Searching for an operational definition of frailty: a Delphi method based consensus statement: the frailty operative definition-consensus conference project. J Gerontol A Biol Sci Med Sci. 2013;68(1):62-7.

27. Malmstrom TK, Miller DK, Simonsick EM, et al. SARC-F: A symptom score to predict persons with sarcopenia at risk for poor functional outcomes. J Cachexia Sarcopenia Muscle. 2017;7(1):28-36. DOI 10.1002/jcsm.12048

28. Ida S, Kaneko R, Murata K. SARC-F for Screening of Sarcopenia Among Older Adults: A Meta-analysis of Screening Test Accuracy. J Am Med Dir Assoc. 2018 (Forthcoming) https://doi.org/10.1016/j.jamda.2018.04.001 


\section{Figure 1. Flow Chart of modified Delphi Method}

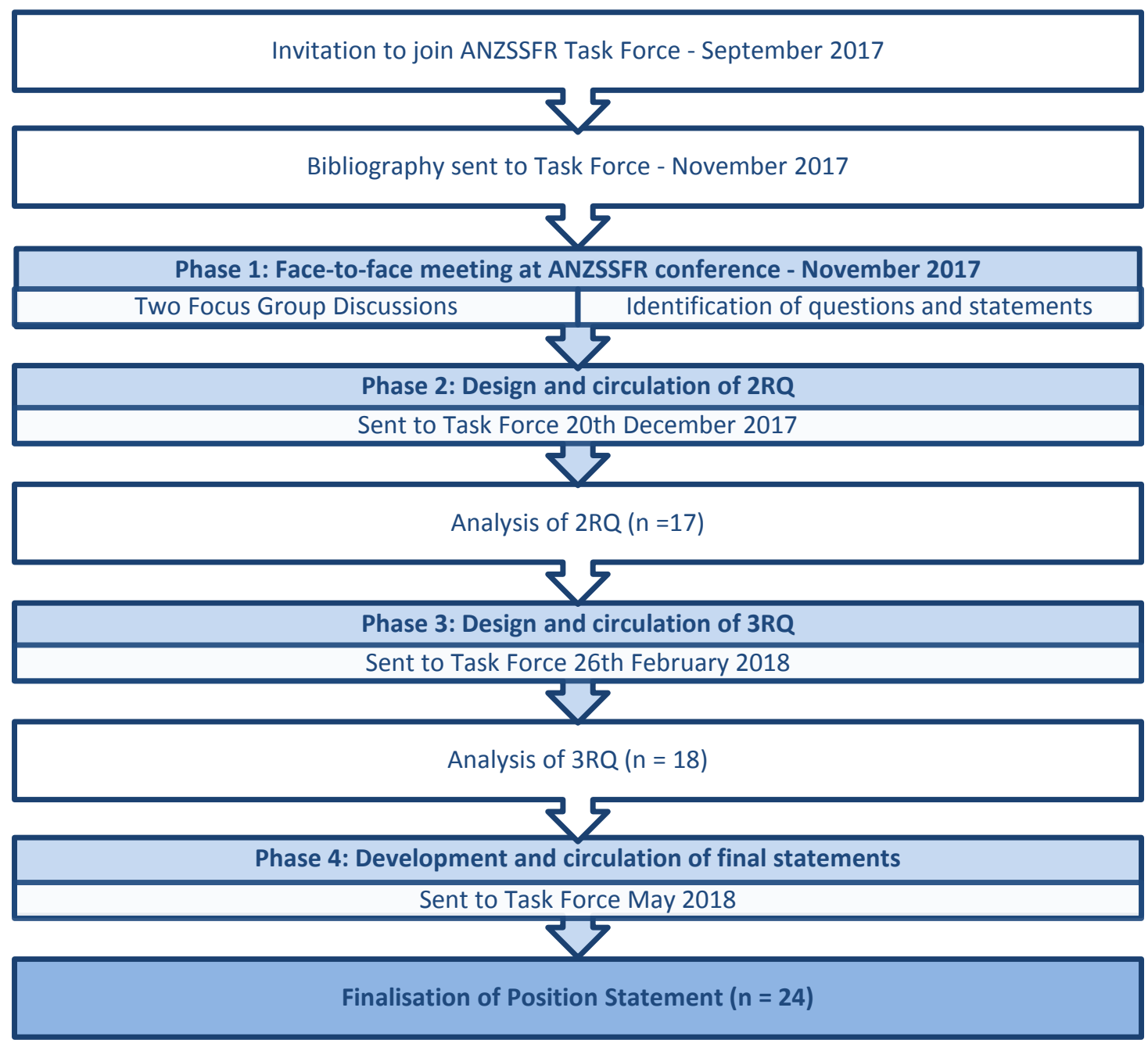

ANZSSFR - Australian and New Zealand Society for Sarcopenia and Frailty Research. $2 R Q$ - Second-round Questionnaire. 3RQ - Third-round Questionnaire. $n=$ number of respondents 
Table 1. Demographics of Task Force members in Phases 2 and 3

\begin{tabular}{|l|c|c|}
\hline Variable & Phase 2 (n = 17) & Phase 3 (n = 18) \\
\hline Mean age, years (SD) & $42.5(10.3)$ & $44.2(10.2)$ \\
\hline Gender, n (Female, \%) & $5(29.4 \%)$ & $6(33.3 \%)$ \\
\hline State or Country (n) & 1 & 1 \\
New South Wales & 10 & 10 \\
Victoria & 1 & 1 \\
Queensland & 2 & 2 \\
South Australia & 1 & 3 \\
Western Australia & 1 & 1 \\
New Zealand & 7 & 10 \\
\hline Position or Role (n) & 2 & 2 \\
Primary clinical researcher & 5 & 4 \\
Post-doctoral researcher & 1 & 1 \\
Physician/geriatrician & 2 & \\
Allied health & & \\
Health service manager & & \\
\hline SD = Standard Deviation & & 1 \\
\hline
\end{tabular}

SD = Standard Deviation 
Table 2. Non-free text questions, statements and respective levels of agreement

\begin{tabular}{|c|c|c|c|}
\hline Questions and Statements & Respondents, n (\% of invitation) & Agreement (\%) & Outcome \\
\hline \multicolumn{4}{|l|}{ Phase 2} \\
\hline $\begin{array}{l}\text { 5. An existing operational definition for sarcopenia should be adopted and } \\
\text { validated using existing Australian and New Zealand data sets }\end{array}$ & $17(73.9 \%)$ & $94.1 \%$ & Accepted \\
\hline $\begin{array}{l}\text { 6. If the adoption of an existing definition is the majority opinion of the Task } \\
\text { Force, which operational definition of sarcopenia do you prefer? } \\
\text { - EWGSOP } \\
\text { - FNIH } \\
\text { - Other }\end{array}$ & $\begin{array}{c}12(52.2 \%) \\
3(13.0 \%) \\
2(8.7 \%)\end{array}$ & $\begin{array}{l}70.6 \% \\
17.7 \% \\
11.8 \% \\
\end{array}$ & Informed $3 R Q$ \\
\hline $\begin{array}{l}\text { 8. If the ANZSSFR proposes to develop a new definition with Aus./NZ data on the } \\
\text { basis of these survey results, please provide suggestions for the proposed } \\
\text { methodology (eg. Measurement tools; cut-points etc.). } \\
\text { - I do not support an additional definition therefore do not wish to comment } \\
\text { - Preferred methodology / cut-points }\end{array}$ & $\begin{array}{l}9(39.2 \%) \\
8(34.8 \%) \\
\end{array}$ & $\begin{array}{l}52.9 \% \\
47.1 \% \\
\end{array}$ & Rejected \\
\hline $\begin{array}{l}\text { 9. Should the society consider recommending the SARC-F as a screening tool for } \\
\text { GPs, allied health and other health professionals? } \\
\text { - Yes } \\
\text { - No }\end{array}$ & $\begin{array}{c}13(56.5 \%) \\
4(17.4 \%)\end{array}$ & $\begin{array}{l}76.5 \% \\
23.5 \%\end{array}$ & Informed $3 R Q$ \\
\hline \multicolumn{4}{|l|}{ Phase 3} \\
\hline $\begin{array}{l}\text { 5. Which operational definition of sarcopenia should the ANZSSFR adopt? } \\
\text { - EWGSOP } \\
\text { - FNIH }\end{array}$ & $\begin{array}{c}17(70.1 \%) \\
1(4.2 \%) \\
\end{array}$ & $\begin{array}{c}94.4 \% \\
5.6 \% \\
\end{array}$ & $\begin{array}{l}\text { Accepted } \\
\text { EWGSOP }\end{array}$ \\
\hline $\begin{array}{l}\text { 6. The ANZSSFR should; } \\
\text { - Adopt and promote an established definition in its entirety (including its } \\
\text { existing cut-points) } \\
\text { - Adopt and promote an established definition and modify this in future if } \\
\text { subsequent validation studies suggest different cut-points are more } \\
\text { appropriate in Australia and New Zealand } \\
\text { - Await the validation studies of Aus./NZ cohorts prior to adopting and } \\
\text { promoting a definition }\end{array}$ & $\begin{array}{c}1(4.2 \%) \\
13(54.2 \%) \\
4(16.7 \%)\end{array}$ & $\begin{array}{l}5.6 \% \\
72.2 \% \\
22.2 \%\end{array}$ & $\begin{array}{c}\text { Informed Phase } \\
4\end{array}$ \\
\hline
\end{tabular}




\begin{tabular}{|c|c|c|c|}
\hline $\begin{array}{l}\text { 7. The pre-specified level of agreement ( } 80 \%) \text { was not reached to adopt the SARC- } \\
\text { F as a recommendation for a screening tool for GPs and other allied health } \\
\text { professionals ( } 77 \%) \text {. To further explore this question, should the ANZSSFR; } \\
\text { - Recommend the SARC-F as a screening tool for GPs, allied health and other } \\
\text { health professionals at the present time } \\
\text { - Not include SARC-F in its recommendations at the present time } \\
\text { - Consider SARC-F in the future when consensus is achieved on the preferred } \\
\text { operational definition of sarcopenia, and research is undertaken to } \\
\text { demonstrate that SARC-F is an acceptable screening tool in Aus./NZ }\end{array}$ & $\begin{array}{c}6(25 \%) \\
1(94.2 \%) \\
11(45.8 \%)\end{array}$ & $\begin{array}{c}33.3 \% \\
5.6 \% \\
61.1 \%\end{array}$ & Rejected \\
\hline \multicolumn{4}{|l|}{ Phase 4} \\
\hline $\begin{array}{l}\text { 1. The ANZSSFR Task Force should promote the EWGSOP definition in our } \\
\text { activities directed at clinicians and researchers. However, a key objective for the } \\
\text { Task Force in future will be to establish appropriate cut-points for appendicular } \\
\text { lean muscle mass, handgrip strength and gait speed in Australian and New } \\
\text { Zealand populations using data from local cohort studies. }\end{array}$ & $23(95.8 \%)$ & $100.0 \%$ & Accepted \\
\hline
\end{tabular}

This is a post-peer-review, pre-copyedit version of an article published in The journal of nutrition, health \& aging. 
Establishing an Operational Definition of Sarcopenia in Australia and New Zealand: Delphi Method Based Consensus Statement

\section{Supplementary Items}

\section{Contents}

Appendix $1 \quad$ Different Operational Definitions of Sarcopenia

Appendix $2 \quad$ Reference list circulated to Task Force prior to Phase 1

Appendix $3 \quad$ Minutes from Phase 1 Face-to-Face Meeting in Adelaide, Australia

Appendix $4 \quad$ Second-round Questionnaire (2RQ)

Appendix $5 \quad$ Third-round Questionnaire (3RQ) 
Appendix 1. Different Operational Definitions of Sarcopenia

\begin{tabular}{|c|c|}
\hline Component & Cut-points \\
\hline \multicolumn{2}{|c|}{ European Working Group on Sarcopenia in Older People [1] } \\
\hline Low muscle mass & $\begin{array}{l}\text { ALM adjusted for height }\left(\mathrm{m}^{2}\right) \text { using whole-body DXA } \\
\text { Men: }<7.26 \mathrm{~kg} / \mathrm{m}^{2} \\
\text { Women: }<5.50 \mathrm{~kg} / \mathrm{m}^{2} \\
\text { SMM adjusted for height }\left(\mathrm{m}^{2}\right) \text { using BIA } \\
\text { Men: }<8.87 \mathrm{~kg} / \mathrm{m}^{2} \\
\text { Women: }<6.42 \mathrm{~kg} / \mathrm{m}^{2}\end{array}$ \\
\hline Low muscle strength & $\begin{array}{l}\text { Hand grip strength using dynamometer } \\
\text { Men: }<30 \mathrm{~kg} \\
\text { Women: }<20 \mathrm{~kg}\end{array}$ \\
\hline Poor physical performance & Gait speed: $\leq 0.8 \mathrm{~m} / \mathrm{s}$ \\
\hline \multicolumn{2}{|c|}{ International Working Group on Sarcopenia [2] } \\
\hline Low muscle mass & $\begin{array}{l}\text { ALM adjusted for height }\left(\mathrm{m}^{2}\right) \text { using whole-body DXA } \\
\text { Men: }<7.23 \mathrm{~kg} / \mathrm{m}^{2} \\
\text { Women: }<5.67 \mathrm{~kg} / \mathrm{m}^{2}\end{array}$ \\
\hline Poor physical performance & Gait speed: $<1.0 \mathrm{~m} / \mathrm{s}$ \\
\hline \multicolumn{2}{|c|}{ Society of Sarcopenia, Cachexia and Wasting Disorders [3] } \\
\hline Low muscle mass & $\begin{array}{l}\text { ALM adjusted for height }\left(\mathrm{m}^{2}\right) \text { using whole-body DXA } \\
<2 \text { SD less than healthy person between age } 20 \text { and } \\
30 \text { years of the same ethnic group }\end{array}$ \\
\hline Poor physical performance & $\begin{array}{l}\text { Gait speed: }<1.0 \mathrm{~m} / \mathrm{s} \text { or }<400 \mathrm{~m} \text { in } 6 \text {-minute walk } \\
\text { test }\end{array}$ \\
\hline \multicolumn{2}{|c|}{ Foundation for the National Institutes of Health [4] } \\
\hline Low muscle mass & $\begin{array}{l}\text { ALM adjusted for BMI }\left(\mathrm{kg} / \mathrm{m}^{2}\right) \text { using whole-body DXA } \\
\text { Men: }<0.789 \\
\text { Women: }<0.512\end{array}$ \\
\hline Low muscle strength & $\begin{array}{l}\text { Hand grip strength using dynamometer } \\
\text { Men: }<26 \mathrm{~kg} \\
\text { Women: }<16 \mathrm{~kg}\end{array}$ \\
\hline \multicolumn{2}{|c|}{ Asian Working Group on Sarcopenia [5] } \\
\hline Low muscle mass & $\begin{array}{l}\text { ALM adjusted for height }\left(\mathrm{m}^{2}\right) \text { using whole-body DXA } \\
\text { Men: }<7.0 \mathrm{~cm}^{2} / \mathrm{m}^{2} \\
\text { Women: }<5.4 \mathrm{~cm}^{2} / \mathrm{m}^{2} \\
\text { SMM adjusted for height }\left(\mathrm{m}^{2}\right) \text { using BIA } \\
\text { Men: }<7.0 \mathrm{~cm}^{2} / \mathrm{m}^{2} \\
\text { Women: }<5.7 \mathrm{~cm}^{2} / \mathrm{m}^{2}\end{array}$ \\
\hline Low muscle strength & Hand grip strength using dynamometer \\
\hline
\end{tabular}


\begin{tabular}{|l|l|}
\hline & $\begin{array}{l}\text { Men: }<26 \mathrm{~kg} \\
\text { Women: }<18 \mathrm{~kg}\end{array}$ \\
Poor physical performance & Gait speed: $\leq 0.8 \mathrm{~m} / \mathrm{s}$ \\
\hline
\end{tabular}

$\mathrm{ALM}=$ Appendicular lean mass. $\mathrm{DXA}=$ Dual X-Ray Absorptiometry. $\mathrm{BIA}=$ Bioimpedence Analysis. SMM = Skeletal Muscle Mass. BMI = Body Mass Index. SD = Standard Deviation

\section{References for Appendix 1}

1. Cruz-Jentoft AJ, Baeyens JP, Bauer JP, et al. Sarcopenia: European consensus on definition and diagnosis: Report of the European working group on sarcopenia in older people. Age Ageing 2010;39(4)412-23. DOI 10.1093/ageing/afq034

2. Fielding RA, Vellas B, Evans WJ, et al. Sarcopenia: An undiagnosed condition in older adults. Current consensus definition: Prevalence, etiology, and consequences. International working group on sarcopenia. J Am Med Dir Assoc 2011;12(4):249-56. DOI 10.1016/j.jamda.2011.01.003

3. Morley JE, Abbatecola AM, Argiles JM, et al. Sarcopenia with limited mobility: An international consensus. J Am Med Dir Assoc 2011;12(6):403-9. DOI 10.1016/j.jamda.2011.04.014

4. Studenski SA, Peters KW, Alley DE, et al. The FNIH sarcopenia project: rationale, study description, conference recommendations, and final estimates. J Gerontol A Biol Sci Med Sci 2014;69(5):547-58. DOI 10.1093/gerona/glu010

5. Chen LK, Liu LK, Woo J, et al. Sarcopenia in Asia: consensus report of the Asian Working Group for Sarcopenia. J Am Med Dir Assoc 2014;15(2):95-101. 


\section{Appendix 2. Reference list circulated to Task Force prior to Phase 1}

1. Rosenberg IH. Summary comments. Am J Clin Nutr. 1989;50(5):1231-3.

2. Baumgartner RN, Romero L, Garry PJ, Heymsfield SB, Koehler KM, Gallagher D, et al. Epidemiology of sarcopenia among the elderly in New Mexico. Am J Epidemiol. 1998;147(8):755-63.

3. Janssen I, Heymsfield SB, Ross R. Low relative skeletal muscle mass (sarcopenia) in older persons is associated with functional impairment and physical disability. J Am Geriatr Soc. 2002;50(5):889-96.

4. Newman AB, Kupelian V, Visser M, Simonsick E, Goodpaster B, Nevitt M, et al. Sarcopenia: alternative definitions and associations with lower extremity function. J Am Geriatr Soc. 2003;51(11):1602-9.

5. Delmonico MJ, Harris TB, Visser M, Park SW, Conroy MB, Velasquez-Mieyer P, et al. Longitudinal study of muscle strength, quality, and adipose tissue infiltration. Am J Clin Nutr. 2009;90(6):1579-85.

6. Visser M, Schaap LA. Consequences of Sarcopenia. Clin Geriatr Med. 2011;27(3):38799.

7. Clark BC, Manini TM. Sarcopenia $\neq$ Dynapenia. J Gerontol A Biol Sci Med Sci. 2008;63(8):829-34.

8. Cooper C, Fielding R, Visser M, van Loon L, Rolland Y, Orwoll E, et al. Tools in the Assessment of Sarcopenia. Calcif Tissue Int. 2013;93(3):201-10.

9. Cruz-Jentoft AJ, Baeyens JP, Bauer JM, Boirie Y, Cederholm T, Landi F, et al. Sarcopenia: European consensus on definition and diagnosis: Report of the European Working Group on Sarcopenia in Older People. Age Ageing. 2010;39(4):412-23.

10. Studenski SA, Peters KW, Alley DE, Cawthon PM, McLean RR, Harris TB, et al. The FNIH Sarcopenia Project: Rationale, Study Description, Conference Recommendations, and Final Estimates. J Gerontol A Biol Sci Med Sci. 2014;69(5):547-58.

11. Biomarkers through consortium. Aging in Motion; [cited 2017]; Available from: http://aginginmotion.org/preliminary-results-sarcopenia-definitions-outcomesconsortium/.

12. Reijnierse EM, de van der Schueren MAE, Trappenburg MC, et al. Lack of knowledge and availability of diagnostic equipment could hinder the diagnosis of sarcopenia and its management. PLOS ONE. 2017;12(10):e0185837.

13. Reijnierse EM, de Jong N, Trappenburg MC, et al. Assessment of maximal handgrip strength: how many attempts are needed? J Cachexia Sarcopenia Muscle. 2017;8(3):466-74.

14. Bijlsma AY, Meskers CG, van den Eshof N, et al. Diagnostic criteria for sarcopenia and physical performance. Age 2014;36(1):275-85.

15. Bijlsma AY, Meskers MC, Molendijk M, et al. Diagnostic measures for sarcopenia and bone mineral density. Osteoporos Int 2013;24(10):2681-91.

16. Bijlsma AY, Meskers CG, Ling CH, et al. Defining sarcopenia: the impact of different diagnostic criteria on the prevalence of sarcopenia in a large middle aged cohort. Age 2013;35(3):871-81.

17. Bijlsma AY, Meskers CG, Westendorp RG, Maier AB. Chronology of age-related disease definitions: osteoporosis and sarcopenia. Ageing Res Rev 2012;11(2):320-4. 


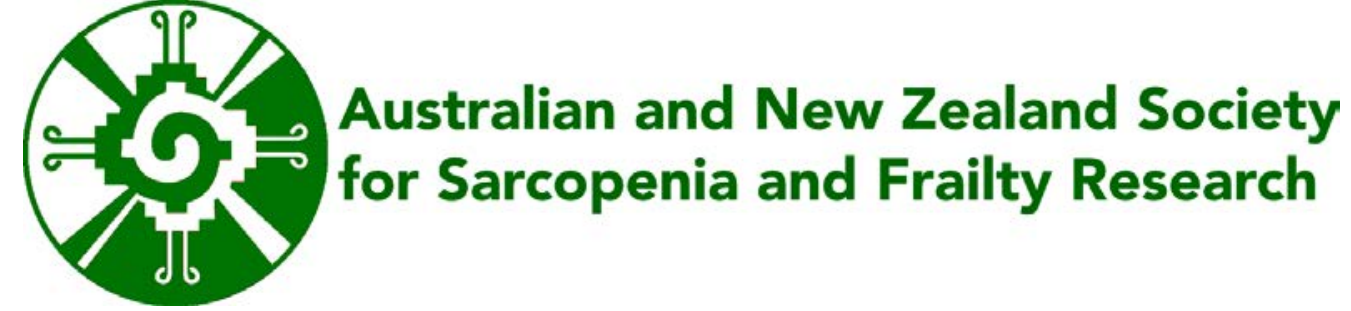

Minutes ANZSSFR Task Force Meeting on Diagnostic Criteria for Sarcopenia.

Sunday November $26^{\text {th }}$, Adelaide Health and Medical Sciences Building, North Terrace, Adelaide.

Present: De-identified

Apologies: De-identified

The primary aim for this Task Force is to determine a single operational definition of sarcopenia for promotion to clinicians and researchers in Australia and New Zealand. We believe this may be achieved through one of the two following strategies:

1. Adoption of an existing international operational definition, measurements and cut-points with research activities including validation of cut-points within Australian older adult cohorts.

2. Development of a new Australian and New Zealand operational definition, measurements or cut-points, the methods for which may or may not be informed by existing international definitions.

Task Force Members will be invited to discuss these potential (and other strategies) and, if a preference is agreed upon, to engage in associated research activities.

\section{Key Discussion Points}

- Do we need a single operational definition of sarcopenia for Australian and New Zealand researchers/clinicians?

- If so, do we support an existing international operational definition (Strategy 1 ) or believe a definition specific to Australia and New Zealand is needed (Strategy 2

Mediator: De-identified: definition of sarcopenia - research setting

Strategy 1: If we are to support an existing operational definition, which should it be? What are the strengths and weaknesses of existing definitions? How do we validate an existing international definition in Australia?

Strategy 2: What work is needed to develop a local operational definition?

What measurements are appropriate and what data is available to support this? There is a challenge in achieving a single "operational Definition" for Sarcopenia. There is not an Aust/ NZ definition for sarcopenia 
NHMRC - comments often came back that we used the wrong definition

To facilitate research and funding

Develop a white paper to publish is JAMDA

Following the definition there were more publications

- RASM (ht2)

- Janssen - normalized by weight

Need consider muscle mass $v$ function

Mass decreases less than function

Dynapenia - Ended up with mass as well as function

EWGSOP - most use this but was meant to be a suggestion

FNIH definition has slightly stricter approach - but may miss a lot of people $<0.8 \mathrm{~m} / \mathrm{sec}$

$\mathrm{FNIH2}$ : Are saying grip strength normalized to $\mathrm{BMI}$ is the most suitable

SARC-F - screening tool (first three questions had greatest AUC)

SPRINNT Trial - combines sarcopenia and frailty definitions

ICD-10-CM: Assignment in 2016.

What is the end point of sarcopenia?

Gait speed?Falls/fractures? Quality of Life?

There is a little overlap in definitions

Assessing muscle mass: DXA v BIA

Differences in ALM controlled for height $v$ BMI

Which is the best indicator of function?

Handgrip strength, gait speed, power?, muscle quality

Muscle wasting $v$ age related sarcopenia

Primary (age-related only) v secondary (bed rest, nutrition)

Strategies : Adopt a current cut off?

Develop our own? Other?

\section{Group discussion}

Is it a problem?

Do we adopt or re-invent?

Sarcopenia definition - is it a definition?

Need an easy tool for diagnosis.

Do we only need one definition?

One definition - clear cut off

Similar one that we can compare to other countries

How do we define the cut off - 2SD below young adults, what do we use as the cut off? Is function the main outcome?

A single operation would be advantageous

Adopting international measures.

Do we need an outcome to work towards to define sarcopenia or the EWGSOP have it in their definition?

Align to an international criteria.

Delphi process to get the best definition (evidence based) and that is put forward to use. Validate the definition in our longitudinal studies 
Maybe have a research definition then a clinical definition

The Delphi process can commence with the task force in a step-wise methodology.

The clinical definition needs to have all the key stake holders involved.

We have international advisors to assist in the process.

Diagnostic criteria: what do clinicians need

Overlap in prevalence is very poor across all the different groups

Frailty and sarcopenia not the same

EWGSOP - suggest use this definition

Had a Sarcopenia road show: Sarcopenia pocket card

\section{Group discussion 1}

Need single definition to be recognised by clinicians

Need an outcome, to test the definitions

Clinician wants an outcome that we are trying to prevent by the diagnosis/treatment.

Sarcopenia has implications for cardiometabolic disease

In addition to inflammation/protein storage. We need clarity in the field.

Receive frequent questions regarding this uncertainty.

Need definition from two perspectives - clinical and research. If ICD-10 approved, have to discuss with Medicare at what rate this diagnosis will be paid etc. If ICD-10 approved, government will want to know how to identify sarcopenia in the acute setting.

Need to establish how to diagnose and what the implications are for making the diagnosis. What is the outcome? What is the intervention to change this outcome/treatment of the condition.

Establish difference for acute and chronic/community etc.

With confusion in the academic literature, this introduces confusion and uncertainty amongst GPs.

We will need our own data to justify the diagnosis.

Clinicians don't know about grip strength. Even though lower limb strength may be a better measure, will be too confusing to introduce new measure

Seconds concerns regarding introducing new measure (lower limb strength). First step is to use/combine existing definitions, and validating this with our own data set.

Issues with gait speed seconded.

FNIH 2 - will be pushing on with developing hand grip strength normalized for BMI

What can you predict with sarcopenia that you cannot predict with gait speed? Do we have evidence that sarcopenia predicts something else not predicted by gait speed? No. We need to find the gaps in sarcopenia research first. Gait speed may predict falls, sarcopenia can predict injury - there are no studies demonstrating this. Delphi process should be utilized in developing white/paper

We should look worldwide for evidence before developing definition in Australia. We should think that the Australian population isn't too different from the rest of the world.

We need the outcome.

No longitudinal prospective study designs have been established.

We have a lot of the data, but no one has looked at it.

Existing Perth data set over large date range. 1500 in data set.

We have data from well-designed longitudinal studies, but we haven't used it.

FNIH 2 correction of adjustment is statistically problematic 
Using only clinical parameters results in can result in a lot of confounding, as opposed to objective measures. We do need an objective measure in addition to the clinical. Useful to have DXA for research, but also gives clues clinically. If there is one adverse outcome associated with a specific finding in the DXA, that can inform discussion with policy makers.

Could an injurious fall then meet the definition of sarcopenia?

Need to prove that there is cost-effectiveness / return of investment on performing DXA/investigations.

\section{Outcomes}

- Align ourselves with international consensus

- Utilization of the Delphi process to develop consensus for the society

o Development of White Paper to answer specific questions (i.e. gait speed in Canada)

o Includes key stakeholders including clinicians, patients, policy makers

o Benefits include external validity, oral and written feedback

o Can develop one paper identify the gaps, second paper showing the outcome of the Delphi process

o This Task force is the first part of the Delphi process.

- We need a key outcome (current gap in the research)

- Need for consensus on research and clinician definition but not necessarily a new definition

0 Reluctance in general for development of an additional definition

- Existing data sets in Aus./NZ should be utilized to answer gaps in the research connecting diagnosis/syndrome with outcome, to validate existing definitions.

- Develop statement for the website by February / grant application time

- Develop paper that identifies the research gaps

- International advisors

\section{Group discussion 2}

What are the priorities for the ANZSSFR in promoting sarcopenia to clinicians and the public? What methods/definitions may be most accessible/relevant to clinicians?

What activities should we engage in to promote sarcopenia assessment and treatment? Are there funding opportunities to support these activities?

What types of clinicians should be targeted?

- $\quad$ Task force to define the guidelines for priority groups (GPs, geriatricians) in Australia

o British Geriatric Society has similar frailty guidelines

o Should have first "success story" - the target group could be the geriatricians

- Incorporate sarcopenia into medical and allied health curricula (but need "runs on the board" first)

- Development of position statement for the society -

- Possibility of incorporating the sarcopenia screen into the GP management plan 70+ screen? - Medicare Benefit Scheme

- Need community health promotion campaign 
- Funding

o Community prevention and advertising approach

o General Practitioner grants for Continuing Medical Education

0 Department of Veterans Affairs through the ANZSSFR

- ANZSGM ASM in June

o Symposium for perioperative medicine

- Other societies to target

o Physio society

o Rehab meetings

- Development of new website for society

Consensus on next steps for the Task Force

1. Develop a societal position on definition of sarcopenia

a. Adopt existing definition

2. Delphi process

a. to commence

3. White Paper

a. For development after Delphi Process

4. Position statement

a. to develop

5. Promotional campaign

a. Health professionals

b. Public

6. Analysis of existing Aus./NZ data sets

a. Definition linking to outcomes

b. Definition agreements

7. Amalgamation of Australian data sets

8. Steps for facilitating communication/discussion

a. Agreement to meet 2 meetings per year Task Force

b. Proposal to have further round table discussions in March/April (face-to-face and online)

9. Increase sectorial awareness

a. Publications in resicare journals

10. Provision of summary statement for the minister on the societies activities

\section{Tasks}

- Delphi process (to set up agenda for future research)

o Research definition

o Clinical definition

- Development of white paper based (include background from Delphi)

- Perform analysis of our data sets (Aus./NZ) to demonstrate agreement with current definitions, and examine for outcome measures

- Agreement in the first session and then move to 2 phases with a panel of experts for (1) RESEARCH and then (2) Clinical with other stake holders.

\section{Presentation}


Presentation of Data of the lack of Guidelines for Sarcopenia. For Medical practitioners, Dieticians, Nurses and Allied Health professionals who at present are all using different ways of diagnosing Sarcopenia (see attached PDF)

In Europe the majority of people thought Sarcopenia and how to maintain muscle health was through exercise and nutrition.

Awareness-ageing, physical acts, malnutrition

Consequences- Falls

Treatment- motivation is needed

Obstacles- 75\% saw no obstacles.

SUMMARY OF PRESENTATION: The need for

One definition

-Diagnostic tools

-Sufficient knowledge to diagnose/manage

- Collaborations between healthcare professionals

-Funding source (ICD-10)

\section{Additional comments noted}

Definitions can be helpful to improve diagnostic reference

Need the correct test or definition to have reliability of diagnosis

First step is to identify the gaps but good to have an initial perspective

To be an International Advisor and mentor

To get an Asian Working Group reprepresentative/advisor.to bring to the Research level a link which is important to Medical Research Future Funds

Learning expectations needs to be defined for all disciplines

Differing levels of diagnostic tools

?integrate into medical Software

Pharmacists are useful at providing additional information

Only $5 \%$ of falls are being referred to the right places.

Can we do earlier screening?

Discussion on Media exposure. Is needed to increase people's awareness of the condition and then it requires Medical education. At this stage there is nothing in the Media.

Ageing is on the agenda throughout Europe, but not in Australia and NZ

The urgent need to place sarcopenia in the teaching/learning and curriculum

Priorities to target: GP's pharmacies, RCGP's send all guidelines through the College to all GP's nationally.

Suggested an initiative to create a smaller taskforce to define the guidelines in Frailty and Sarcopenia, for all GPs in the country. It must be simple. Target the overall population.

Geriatricians to assess the condition when referred on, but must have GP's aware in order to refer on. Need a success story in Australia. The priority to train and provide GP's, or integrate Sarcopenia into practices, or include in Geriatrician's curriculum OR target all.

Need pathways and need investment to get into all the pathways. 
Already have Falls and Fracture clinics, and Gait and Balance. Can Sarcopenia be added/integrated into it?

Curriculum difficult to get into. Sarcopenia needs a profile. ?AJA Journal/Aust Society

Geriatricians?

To take initiative on this area

Short term goals GP's are crucial to start with.

Link falls and fractures to muscle and this will profile with Sarcopenia.

Need to start with a positive statement which gives it more power.

Need to focus of Sarcopenia and keep Osteoporosis out of it.

Consensus was that it was better to start the target with Geriatricians and then lead onto GP's

$70+$ Screen? Can a screen for Sarcopenia be incorporated into this screening and also Gait speed?

A preventative message needs to be circulated

Use of work Sarcopenia. Geriatricians keep sending it back to GP's and then they may

question ad enquire what it is?

National Sarcopenia Day?

\section{Funding Questions:}

Issue can be relatively easily funded. Target colleagues for small grants, that is educational grants.

From all tiers of Government and ask all to do some groundwork

ANZGM ask to incorporate into new social development. Annual Conference in June and will be involved.

? ANZSSFR stand at the Geriatric meeting in June?

Need to target all related Societies and their meetings/conferences

Website: critical information. Website being established at this time.

\section{AGREEMENT AT THE END OF THIS MEETING.}

1.take position as a society on which definition to recommend with the preferenceadopting what is already put there.

2.Delphi process the first step to start

3. position statement to coincide with public awareness, conferences early/mid 2018

4.data together with etc. and comparisons made

5.achieve a conversation on strategies to reach a consensus

6.invitations for involvement in activities

7.white paper

8. cohort synthesis and data analysis

9. prominent activities

10. reconvene next ANZSSFR Annual meeting 2018

11. early meetings March/April; with some smaller meetings of 5-6 people

Ended the meeting and thanked everyone for making the time to attend. Agreed it was a most productive exercise

\section{"We want to present a case to the world."}


MEETING CONCLUDED AT 12.30PM 


\section{Appendix 4. Second-round Questionnaire (2RQ)}

1. What is your age?

2. What is your gender?

3. Please select your state and country

4. What is your Position / job title /role?

\section{Quantification and confirmation of focus group discussion results}

5. Clinical operational definition for sarcopenia.

Select the statement that reflects your view;

a. An existing operational definition for sarcopenia should be adopted and validated using existing Australian and New Zealand data sets

b. A new Australian and New Zealand operational definition for sarcopenia should be developed using existing Australian and New Zealand data sets

c. Other - free text.

6. If the adoption of an existing definition is the majority opinion of the Task Force, which operational definition of sarcopenia do you prefer;

a. European Working Group on Sarcopenia in Older People (EWGSOP)

b. Foundation for the National Institutes of Health Sarcopenia Project (FNIH)

c. Other - free text

7. Please give your reasoning behind your selection in 2 - Free text

8. If the ANZSSFR proposes to develop a new definition on the basis of these survey results, please provide suggestions for the proposed methodology (eg. Measurement tools; cut-points etc.). - Free text

9. The SARC-F is a 5 question self-rated screening questionnaire for sarcopenia. Should the society consider recommending the SARC-F as a screening tool for GPs, allied health and other health professionals?

a. Yes

b. No

Further comment

10. Should the ANZSSFR adopt distinct definitions, with one for clinical purposes and one for research purposes?
c. Yes

d. No

If you answered a, which definitions should be used for each? Free text.

11. In addition to the establishment of an operational definition, what are the key knowledge gaps on which sarcopenia research should focus? Free text

12. Are you interested in contributing further to the development of the White Paper on Sarcopenia? (In subsequent phases your email address will be requested).

- Yes

- No

13. Any further comments you wish to make? Free text 


\section{Appendix 5. Third-round Questionnaire (3RQ)}

Participant demographic details

1. What is your age in years?

2. What is your gender?

3. In which state or country are you based?

4. What is your job title / role (Physician/Specialty, Researcher, Allied Health Professional, other)?

5. Phase II of the Delphi conference of the ANZSSFR Task Force has demonstrated agreement (approx. 94\%) for the adoption of an existing operational definition for sarcopenia in Australia and New Zealand. We now hope to achieve a consensus (80\% agreement) on which existing operational definition should be adopted. Which operational definition of sarcopenia should the ANZSSFR adopt?

e. European Working Group on Sarcopenia in Older People (EWGSOP)

f. Foundation for the National Institutes of Health Sarcopenia Project (FNIH)

6. The ANZSSFR Task Force has voted to adopt an established operational definition of sarcopenia. However, cut-points for sarcopenia components in existing definitions have previously been modified in different geographic locations (for example the Asian Working Group on Sarcopenia adopted the EWGSOP algorithm for sarcopenia case-finding but applied cut-points that may be more appropriate in Asian populations).

The ANZSSFR Task Force intends to undertake validations studies using the chosen definition in Aus./NZ data sets over the next 18 months.

At the present moment, do you think the ANZSSFR should;

A. Adopt an established definition in its entirety (including existing cut-points)

B. Adopt and promote an established definition and modify in future if subsequent validation studies suggest different cut-points are more appropriate in Australia and New Zealand

C. Await the validation studies of Aus./NZ cohort prior to adopting and promoting a definition

Question 7-The pre-specified level of agreement (80\%) was not reached to adopt the SARC-F as a recommendation for a screening tool for GPs and other allied health professionals (77\%). To further explore this question, should the ANZSSFR;

A. Recommend the SARC-F as a screening tool for GPs, allied health and other health professionals at the present time

B. Not include SARC-F in its recommendations at the present time

C. Consider SARC-F in the future when consensus is achieved on the preferred operational definition of sarcopenia, and research is undertaken to demonstrate that SARC-F is an acceptable screening tool in Aus./NZ 
This is a post-peer-review, pre-copyedit version of an article published in The journal of nutrition, health \& aging The final authenticated version is available online at: http://dx.doi.org/10.1007\%2Fs12603-018-1113-6 